\title{
Systematic analysis of actinide decay heat focused on the mixed oxide fuel
}

\author{
N. Hagura ${ }^{1, a}$, T. Yoshida ${ }^{1}$, and T. Nakagawa ${ }^{2}$ \\ 1 Musashi Institute of Technology, 1-28-1 Tamazutsumi, Setagaya-ku, Tokyo 158-8557, Japan \\ 2 Nuclear Data Center, Japan Atomic Energy Agency, 2-4 Shirane, Shirakata, Tokai-mura, Naka-gun, Ibaraki 319-1195, Japan
}

\begin{abstract}
Radioactivity and decay power of spent MOX fuel are very crucial in term of storage or disposal. In this work, the uncertainty of actinide decay heat introduced by the uncertainty of nuclear data and the prediction accuracy of isotopic generation was studied. 100 year after discharge, for example, a 5\% error on the decay heat will be introduced by a $20 \%$ uncertainty on the Pu-240 capture cross section through Am-241 production. We performed sensitivity analyses with covariance data in JENDL-3.3 of cross sections of notable nuclides on each energy group. As an example, standard deviation of the calculated actinide decay heat 100 year after discharge is evaluated to be $1.26 \%$ on a $1 \sigma$ base.
\end{abstract}

\section{Introduction}

Nowadays, nuclear power plants in the world accumulate a vast amount of spent fuel. Until a few years ago, the concept of once-through was supported by some countries because the diffusion of the reprocessing technique, which is possible to be converted to military application, was a serious menace. There is, however, a limit on disposal space of spent nuclear fuels. Hence the countries that supported the once-through concept are returning the policy to the reprocessing including an advanced nuclear fuel cycle. Japan, where the resource is scarce, has stuck to the way that the spent fuel is reprocessed and is not disposed directly.

Under this background, the Mixed OXide (hereafter MOX) fuels will play a role of greater importance in the future. It is, then, essential to understand the characteristics of spent MOX fuels. Especially it is important to evaluate actinide decay heat appropriately, since the spent MOX fuels contain more actinide nuclides than spent uranium fuels.

In the present paper, the uncertainty on the actinide decay heat calculation introduced by the uncertainty on nuclear data and the prediction accuracy of isotopic generation is described.

100 year after discharge, for example, a $7 \%$ error on the decay heat will be introduced by a $10 \%$ uncertainty in the $\mathrm{Pu}-$ 241 generation calculation.

We tried to perform sensitivity analyses with covariance data in JENDL-3.3. The solid analyses with covariance data are necessary to satisfy the demand of higher reliability. Therefore the importance of the evaluation that uses covariance data will rise more and more in the future. In this work, the covariance data are applied to actinide decay heat calculations.

\section{Procedure of analyses}

In this study, we made use of the SWAT code system ${ }^{1}$ developed by Japan Atomic Energy Research Institute (JAERI, JAEA at present) and the ORIGEN2 code [2] developed by

a Presenting author, e-mail: naoto_hagura@hotmail.com
Oak Ridge National Laboratory (ORNL). Burn-up calculation was performed by the SWAT code, and decay heat calculation was carried out by the ORIGEN2 code.

In SWAT, typical PWR pin cell model was selected as calculation model. Standard component of MOX fuel was specified as PWR60G-MOX ${ }^{3}(7.9 \mathrm{wt} \%$ Pu-enriched and $0.23 \mathrm{wt} \%$ depleted uranium). Calculation conditions are as follows: Thermal power is set to $37.9 \mathrm{MW} / \mathrm{t}$, discharged burnup is $60 \mathrm{GWd} / \mathrm{t}$, and the library used is JENDL-3.3. Isotopic inventories calculated by SWAT were substituted into the input of ORIGEN2 code. For comparison, we also dealt with other fuel types: PWR33G-MOX [3] and PWR60G-UO2 [3].

On sensitivity analyses, the variance $\mathrm{V}$ is obtained by the following equation

$$
\mathrm{V}=\mathrm{GMG}^{\mathrm{t}}
$$

where $\mathrm{G}$ means the sensitivity coefficient at each cooling time, $\mathrm{G}^{\mathrm{t}}$ stands for the transpose matrix of $\mathrm{G}$, and $\mathrm{M}$ stands for the covariance matrix. We described them in a 21 energy-group structure. Moreover $\mathrm{G}$ is given by the following equation:

$$
G=\frac{\frac{\Delta D H}{D H}}{\frac{\Delta \sigma}{\sigma}} .
$$

Covariance data were obtained by processing JENDL-3.3 by the ERRORJ code [4]. Figure 1 shows the correlation matrix of the Am-241 capture. Figure 2 shows the relative standard deviation of the Am-241 cross section.

\section{Calculation results}

\subsection{Spent fuel decay heat in perspective}

First of all, for keeping the decay heat and radioactivity of spent fuel in perspective, we show the results of standard calculation of this study in figure 3. On the figure, thin solid line stands for the actinide component (ACT) of decay heat. We pay attention to this ACT. The difference that exceeds one decade is seen between PWR60G-MOX and PWR60G-UO2 in the actinide component just after discharge ( 0.1 year). As a 


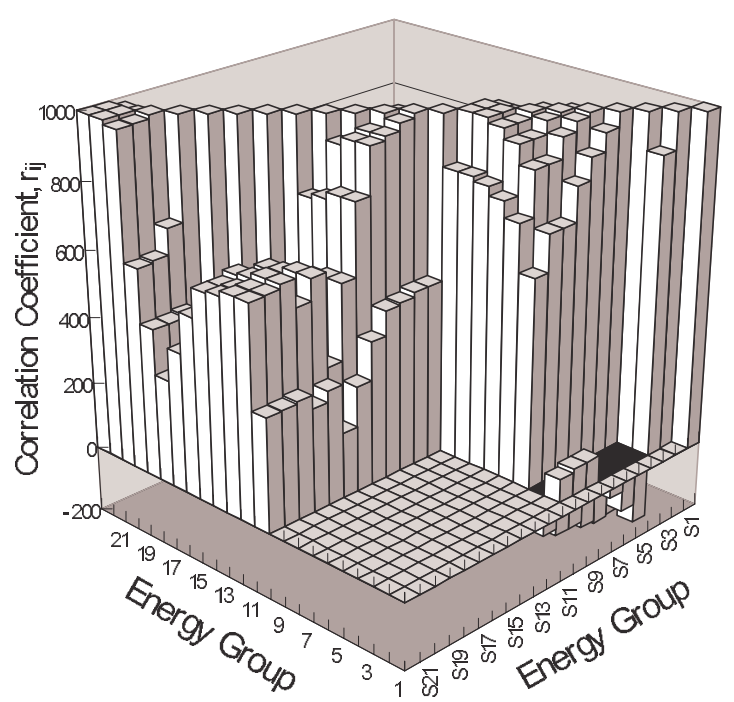

Fig. 1. Correlation matrix of the Am-241 capture.

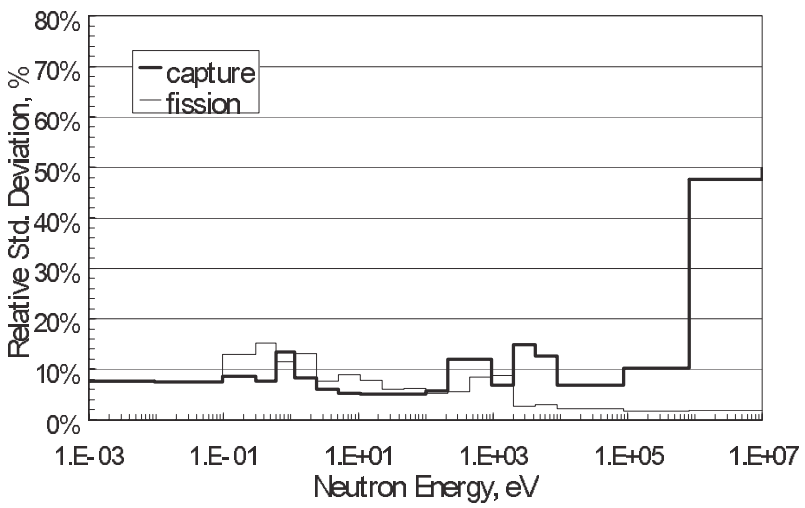

Fig. 2. Relative standard deviation of Am-241 in JENDL-3.3.

result, total decay heat $(\mathrm{ACT}+\mathrm{FP})$ is raised for high burn-up MOX.

In each cooling time, the nuclides that have dominant contribution are as follows (cooling time): Cm-242 ( 1 yr), $\mathrm{Pu}-238$ ( $100 \mathrm{yr})$, Am-241 ( 500 yr), Pu-240 ( 104 yr), $\mathrm{Pu}-239\left(\sim 10^{5} \mathrm{yr}\right)$, and nuclides of neptunium series $\left(>10^{5} \mathrm{yr}\right)$.

\subsection{Impact of the prediction accuracy of isotopic generation}

On several PIE analyses, there are discrepancies in the $\mathrm{Pu}$ vector and minor actinides (MAs) inventories that cannot be ignored. $\mathrm{Pu}$ isotopes have a few percent disagreement and that MAs have tens of percent disagreement [5].

Therefore, evaluation of decay heat including their uncertainty is required. Figure 4 shows the ratio of difference of total decay heat by introducing uncertainty of the burn-up calculation results on $\mathrm{Pu}-241$. The uncertainty in the $\mathrm{Pu}-241$ inventory at the end of irradiation is set to $2 \%-20 \%$.

This figure indicates that a $7 \%$ error on the total decay heat 100 year after discharge is caused by a $10 \%$ uncertainty on the $\mathrm{Pu}-241$ generation calculation results. And then the figure shows the variations of decay-heat behavior with respect to

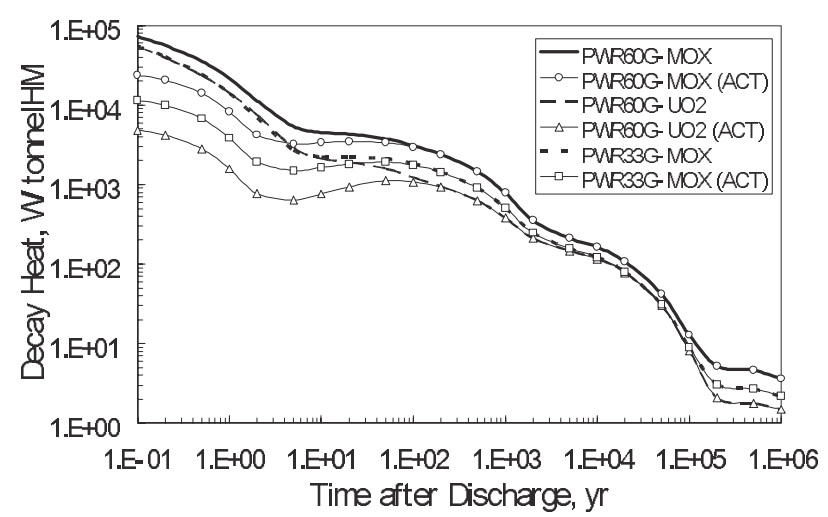

Fig. 3. Total decay heat and actinide component of spent fuel.

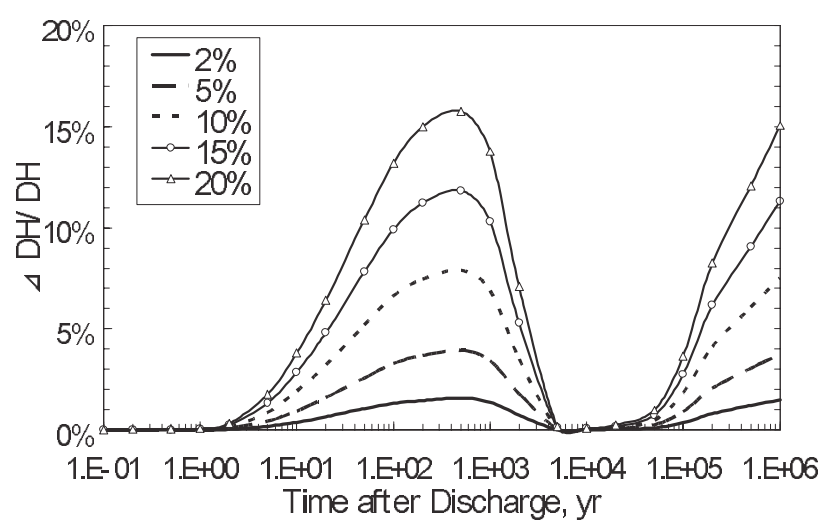

Fig. 4. Uncertainty of the FP and actinide decay heat introduced by the prediction accuracy of isotopic generation of Pu-241.

the intentional change in the discharge inventory of Pu-241 isotope. The peak of around 500 year is not coming directly from $\mathrm{Pu}-241$ itself but through Am-241. Pu-241 decays into Am-241 with the half-life of 14.35 years. It is $\mathrm{Pu}-241$ that dominates the amount of Am-241 long after discharge. Am241 is an important nuclide which dominates the decay heat for an important period of storing spent fuels from ten-odd years to thousands of years.

\subsection{Impact of the uncertainty of one-group cross section}

On the spent fuel storage term (from 10 years to several 100 years), Am-241 is the dominant nuclide. Importance of the amount of $\mathrm{Pu}-241$ has already been described. At reactor shutdown, the inventory of Pu-241 is remarkable. Therefore, it is necessary to assess the reaction of parent nuclides of Pu241, such as $\mathrm{Pu}-240$. The influence on total decay heat was examined, when capture cross section of $\mathrm{Pu}-240$ was changed from 10 to 50 percent (fig. 5).

Figure 5 indicates that a 5\% error on the total decay heat is caused by a $20 \%$ uncertainty on the Pu-240 capture cross section. And then the figure shows some peaks for all period. The peak of around 500 year means the difference originated by Am-241. The dip of about $10^{4}$ year results from reduction of $\mathrm{Pu}-240$. Around $10^{6}$ year, nuclides of the neptunium series are dominant. 


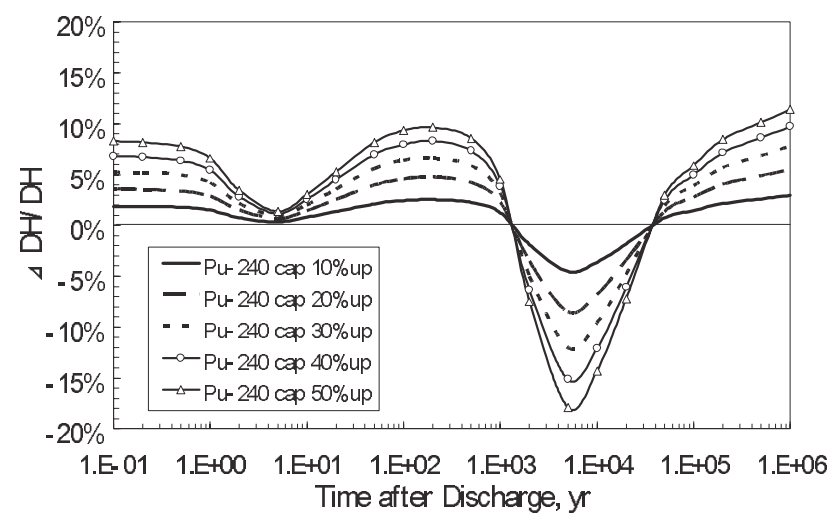

Fig. 5. Uncertainty of the total decay heat introduced by the uncertainty of the one-group capture cross section of Pu-240.

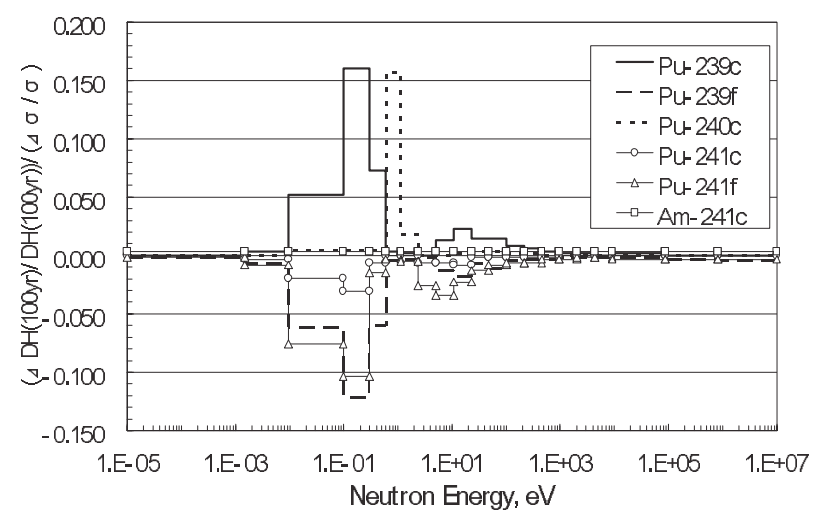

Fig. 6. Sensitivity coefficient of the total decay heat $100 \mathrm{yr}$ after discharge.

Above we dealt with one-group constant and discussed in an approximate way of change of decay heat to appear. Hereafter it is examined including sensitivity coefficients with 21 energy-group structure.

\subsection{Multi-group sensitivity analysis with covariance data}

Covariance data of 32 nuclides including 13 actinides are stored in JENDL-3.3. We tried to apply these data to calculation of actinide decay heat and evaluate their standard deviation originated by nuclear data uncertainty. We evaluated them in a 21 energy-group structure. In ref. [6], reactor performance parameters that use a similar way of sensitivity analysis with covariance matrices is assessed.

Figure 6 shows the sensitivity coefficients of each nuclide and reaction on ACT hundred years after discharge. Since thermal reactor with MOX fuel is selected, high sensitivities are concentrated at epithermal region. Higher sensitive reactions are capture in $\mathrm{Pu}-239$ and $\mathrm{Pu}-240$, and fission in $\mathrm{Pu}-239$ and $\mathrm{Pu}-241$.

Table 1 summarizes standard deviation of each nuclide and reaction. The table indicates dominant nuclides are $\mathrm{Pu}-239$, $\mathrm{Am}-241$, and $\mathrm{Pu}-238$. As a result, standard deviation in this case is evaluated to be $1.26 \%$ according to the equations (1) and (2).
Table 1. Decay heat uncertainties 100 year after discharge coming from each nuclide and reaction.

\begin{tabular}{cccc}
\hline & cap & fis & Total \\
\hline $\mathrm{Np}-237$ & 0.0000 & - & 0.0000 \\
$\mathrm{Pu}-238$ & 0.0037 & - & 0.0037 \\
$\mathrm{Pu}-239$ & 0.0077 & 0.0080 & 0.0111 \\
$\mathrm{Pu}-240$ & 0.0016 & - & 0.0016 \\
$\mathrm{Pu}-241$ & 0.0010 & 0.0015 & 0.0019 \\
$\mathrm{Am}-241$ & 0.0039 & - & 0.0039 \\
$\mathrm{Cm}-242$ & 0.0000 & - & 0.0000 \\
\hline Total & 0.0096 & 0.0082 & $1.26 \%$ \\
\hline
\end{tabular}

Table 2. Standard deviation of each condition and fuel type 100 year after discharge ( $1 \sigma$ basis)

\begin{tabular}{cccc}
\hline & PEC & FEC & NC \\
\hline PWR60G-MOX & $1.26 \%$ & $2.15 \%$ & $1.01 \%$ \\
PWR60G-UO2 & $3.29 \%$ & $7.80 \%$ & $3.00 \%$ \\
PWR33G-MOX & $1.13 \%$ & $1.83 \%$ & $0.94 \%$ \\
\hline
\end{tabular}

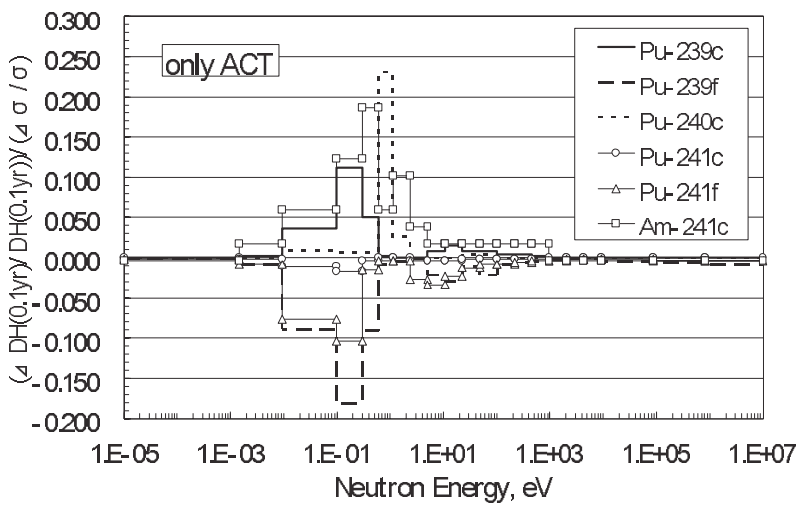

Fig. 7. Sensitivity coefficient of the total decay heat $0.1 \mathrm{yr}$ after discharge (actinide component only).

Table 2 shows difference among partial energy correlation (PEC), full energy correlation (FEC), and no correlation (NC) results. Moreover, this table shows difference of fuel type and discharged burn-up. The result indicates that the relation between the 3 values is FEC > PEC > NC on any fuel type. The difference between PEC and others appears in PWR60GMOX though it is not more remarkable than PWR60G-UO2. Then, it is valuable to consider the contribution of nondiagonal elements.

Short after the discharge of spent fuel, or within several years, total decay heat is dominated by fission products. While in this area FP component should be remarked in the engineering fields, authors also think it is important to evaluate the decay heat of actinide component appropriately.

Figure 7 shows the sensitivity coefficient of each nuclide and reaction 0.1 year after discharge. The sensitivity of capture of Am-241 that differs from figure 6 is large. In this cooling time region, main contributor of actinide component is Cm242. Therefore parent nuclides of Cm-242, such as Am-241, indicate higher sensitivity. Table 3 shows standard deviation of PEC is $4.12 \%$ of this MOX fuel case. 
Table 3. Standard deviation of the calculated actinide decay heat 0.1 year after discharge.

\begin{tabular}{ccc}
\hline \multicolumn{3}{c}{ PWR60G- MOX } \\
\hline PEC & FEC & NC \\
\hline $4.12 \%$ & $5.69 \%$ & $2.54 \%$ \\
\hline
\end{tabular}

\section{Conclusion}

It is important to evaluate appropriately the characteristics of spent MOX fuels, especially decay heat. In this study, sensitivity analyses of prediction accuracy of isotopic generation and uncertainty of cross sections were performed. Between 100 and 1000 years after discharge of the spent MOX fuel, the total decay heat is dominated by Am-241. We indicated to pay attention to the generation of $\mathrm{Pu}-241$ which decays into Am241. The amount of $\mathrm{Pu}-241$ has a close relation to the capture cross sections of $\mathrm{Pu}-239$ and $\mathrm{Pu}-240$. The uncertainty of the burn-up calculation is discussed. At 100 year after discharge, for example, a $7 \%$ error on the total decay heat 100 year after discharge is caused by a $10 \%$ uncertainty on the $\mathrm{Pu}-241$ generation calculation results. And a 3\% error on the total decay heat is caused by a $10 \%$ uncertainty on the Pu- 240 capture cross section.

In the sensitivity analysis in multi-group scheme with covariance data, standard deviation is obtained by matrix calculation with sensitivity coefficient and covariance matrix. As a result, standard deviation in this study is evaluated to be $1.26 \%$ ( $1 \sigma$ basis). A sizable difference between partial energy correlation (PEC) and no correlation appears in PWR60GMOX though it is not more remarkable than PWR60GUO2. Then, authors thought it is valuable to consider the contribution of non-diagonal elements. On short term after the discharge of spent fuel, parent nuclides of $\mathrm{Cm}-242$, such as Am-241, indicate higher sensitivity. As a result, standard deviation of PEC is evaluated to be $4.12 \%$ in the present case.

\section{References}

1. K. Suyama, T. Kiyosumi, H. Mochizuki, JAERI-Data/Code 2000027, JAERI (2000) (in Japanese).

2. A.G. Croff, ORNL/TM-7175, Oak Ridge National Laboratory (1980).

3. Y. Ando, H. Takano, JAERI-Research 99-004, JAERI (1999) (in Japanese)

4. G. Chiba, M. Ishikawa, Proc. of Int. Conf. on Nucl. Data for Sci. and Tech., Santa Fe, USA, 2005, pp. 468-471.

5. Y. Ando, K. Yoshioka, I. Mitsuhashi, K. Sakurada, S. Sakurai, Proc. of the 7th International Conference on Nuclear Criticality Safety, Tokai, Japan, Oct. 20-24, 2003 (2003), pp. 494-499.

6. G. Aliberti et al., Proc. of the Int. Workshop on Nucl. Data Needs for Gen. IV Nucl. Energy Systems, Antwerp, Belgium, Apr. 5-7, 2005 (2005). 